\title{
Structure-aware Linear Solver for Realtime Convex Optimization for Embedded Systems
}

DOI:

10.1109/LES.2017.2700401

\section{Document Version}

Accepted author manuscript

Link to publication record in Manchester Research Explorer

\section{Citation for published version (APA):}

Yamazaki, I., Nooshabadi, S., Tomov, S., \& Dongarra, J. (2017). Structure-aware Linear Solver for Realtime Convex Optimization for Embedded Systems. IEEE Embedded Systems Letters.

https://doi.org/10.1109/LES.2017.2700401

\section{Published in:}

IEEE Embedded Systems Letters

\section{Citing this paper}

Please note that where the full-text provided on Manchester Research Explorer is the Author Accepted Manuscript or Proof version this may differ from the final Published version. If citing, it is advised that you check and use the publisher's definitive version.

\section{General rights}

Copyright and moral rights for the publications made accessible in the Research Explorer are retained by the authors and/or other copyright owners and it is a condition of accessing publications that users recognise and abide by the legal requirements associated with these rights.

\section{Takedown policy}

If you believe that this document breaches copyright please refer to the University of Manchester's Takedown Procedures [http://man.ac.uk/04Y6Bo] or contact uml.scholarlycommunications@manchester.ac.uk providing relevant details, so we can investigate your claim.

\section{OPEN ACCESS}




\title{
Structure-aware Linear Solver for Realtime Convex Optimization for Embedded Systems
}

\author{
Ichitaro Yamazaki, Saeid Nooshabadi, Senior Member, IEEE, Stanimire Tomov, and Jack Dongarra.
}

\begin{abstract}
With the increasing sophistication in the use of optimization algorithms such as deep learning on embedded systems, the convex optimization solvers on embedded systems have found widespread use. This letter presents a novel linear solver technique to reduce the run-time of convex optimization solver by using the property that some parameters are fixed during the solution iterations of a solve instance. Our experimental results show that the run-time can be reduced by two orders of magnitude.
\end{abstract}

Index Terms-Realtime embedded convex optimization solver, KKT

\section{INTRODUCTION}

Recent advances in convex optimization [1] [2] have enabled their use as realtime solvers for embedded systems [3] [4]. Embedded solvers come with certain features that can be exploited to reduce the complexity of the design. For instance, in many cases, the embedded solvers only require a low accuracy. One such example is the model predictive control (MPC), where very low accuracy is needed to obtain acceptable control performance [5]. Another feature is that, for an embedded solver, the structure of the problem does not change from one solve to the next. For example, for a given problem instance of Kalman filtering, an embedded solver perform many solves, where the dimensions and structure of the system state, input and output vectors, and steady-state error covariance matrix are all fixed, and the system parameters remain unchanged with each realtime solution iteration. Furthermore, the change in the numerical values of the solver parameters between two subsequent instances of the problem is usually small. The fixed size and structure of the system provides an opportunity to design and optimize the solver at the development time and to significantly reduce the solve time.

To obtain high performance on the embedded system, recent tools such as CVXGEN code generator [6] and ECOS [7] provide frameworks for realtime convex optimization solvers. There are also some attempts in code generation for smallsized basic linear algebra opeations like vector-matrix multiplication [8]. CVXGEN takes a high-level description of the optimization problem, employs the CVX technique of disciplined convex programming (DCP) [9] to ensure convexity, and then generates a flat, and library-free $\mathrm{C}$ code. The

Saeid Nooshabadi is with the Department of Electrical and Computer Engineering, Michigan Tech, Houghton, MI, e-mail:\{saeid\}@mtu.edu; Ichitaro Yamazaki, Stanimire Tomov and Jack Dongara are with Innovative Computing Laboratory (ICL), the University of Tennessee, Knoxville, e-mail\{iyamazak, tomov, dongarra\}@icl.utk.edu

Manuscript received January 2017; revised: April 2017 generated code can be compiled into a high performance solver for the specific problem family (e.g., all the matrices have the same sparsity structure). Though CVXGEN solver obtains a high performance that meets the strict realtime constraint enforced on the solution time, the size of the problem is limited to 100 or so variables for the code generator to successfully generate a flat loop-free $\mathrm{C}$ code.

In DCP, a quadratic programming (QP) convex problem is transformed into a standard form that seeks for the vector variable $v$ that minimizes the following optimization problem [10],

$$
\begin{array}{lc}
\text { minimize } & (1 / 2) v^{\mathrm{T}} Q v+q^{\mathrm{T}} v \\
\text { subject to } & G v \preceq h \text { and } A v=b,
\end{array}
$$

where $v, q \in \mathbf{R}^{n}, Q \in \mathbf{S}_{+}^{n} \succeq 0$ (a square symmetric positive semidefinite matrix), $A \in \mathbf{R}^{m \times n}, G \in \mathbf{R}^{p \times n}, b \in \mathbf{R}^{m}$, and $h \in \mathbf{R}^{p}$. At each iteration of the solve instance, most of the time is spent solving a family of Karush Kuhn Tucker (KKT) [2] linear system of equations $\widehat{K} \widehat{x}=\widehat{c}$, whose coefficient matrices $\widehat{K}$ all have the following block structure:

$$
\widehat{K}=\left(\begin{array}{cc|cc}
Q & 0 & G^{\mathrm{T}} & A^{\mathrm{T}} \\
0 & S^{-1} Z & I_{p} & 0 \\
\hline G & I_{p} & 0 & 0 \\
A & 0 & 0 & 0
\end{array}\right),
$$

where $(.)^{\mathrm{T}}$ denote the matrix transpose, $I_{p} \in \mathbf{R}^{p \times p}$ is the identity matrix, $S=\operatorname{diag}(s) \in \mathbf{R}^{p \times p}$ and $Z=\operatorname{diag}(z) \in \mathbf{R}^{p \times p}$ are diagonal matrices, and $s \in \mathbf{R}^{p}$ and $z \in \mathbf{R}^{p}$ are vectors representing the slack variables and inequality multipliers, respectively, in the KKT condition [11]. The optimization solver performs several iterations until a pre-determined accuracy or the maximum number of iterations is reached.

To solve the linear system, CVXGEN first computes the $\mathrm{LDL}^{\mathrm{T}}$ decomposition of the KKT matrix $\widehat{K}$, i.e., $P \widehat{K} P^{\mathrm{T}}=$ $\widehat{L} \widehat{D} \widehat{L}^{\mathrm{T}}[12]$, where $P$ is a permutation matrix, $\widehat{L}$ a lower triangular matrix with unit diagonals, and $\widehat{D}$ is a diagonal matrix. Then, the solution $\widehat{x}$ is computed through the sequence of forward substitution, scaling, and backward substitution.

The current embedded optimization solvers, while good at taking advantage of the structure of the problem family (e.g. sparsity), fails to take advantage of the fact that several blocks of the KKT matrix are fixed during the iterations of a given solve instance. In most practical signal processing applications for embedded systems, such as linearizing pre-equalizer, Kalman filtering, sliding window smoothing or estimation [13], only the vectors $q, h$ or $b$ in (1) change from one solve 
instance to the next. These vectors only affect the right-handside vector $\widehat{c}$ of the KKT linear system. In the online array weight design for adaptive array signal processing [3], only the matrix $G$ changes between the solve instances. In many other problems of interests, only the diagonal block $S^{-1} Z$ changes at each solve instance. This letter proposes to reduce the time to solve the family of the KKT linear systems by exploiting the fact that several blocks in the KKT matrix $\widehat{K}$ are fixed during the solution iterations of a given problem instance.

\section{AlgorithmS}

We consider two types of changes in the KKT matrix for the interior-point methods [1] [2]: A) the change that occurs at each iteration of a solve instance and B) the change that persists across all the solution iterations in one solve instance. Hence, our linear solver consists of three phases: 1) off-line setup which is done once for all the solve instances of a problem family, 2) off-line update which is done once for each solve instance, and 3) on-line factorization and solve which are done once at each iteration. To avoid factorizing singular blocks, like CVXGEN, we regularize $\widehat{K}$ using the standard techique [11].

\section{A. Fixed $Q, A, G$ and variable $S^{-1} Z$}

Between the solution iterations, it is often that only the diagonal block $S^{-1} Z$ changes. We take advantage of this property and amortize the cost of the operations that involve $Q, A$ and $G$ over all the solve instances. For this, we solve an equivalent, implicitly-reordered, linear system, $K x=b$, i.e.,

$$
\left(\begin{array}{c|c|c|c}
Q & A^{\mathrm{T}} & 0 & G^{\mathrm{T}} \\
\hline A & 0 & 0 & 0 \\
\hline 0 & 0 & S^{-1} Z & I_{p} \\
\hline G & 0 & I_{p} & 0
\end{array}\right)\left(\begin{array}{c}
\widehat{x}_{1} \\
\hline \widehat{x}_{4} \\
\hline \widehat{x}_{2} \\
\hline \widehat{x}_{3}
\end{array}\right)=\left(\begin{array}{c}
\widehat{c}_{1} \\
\hline \widehat{c}_{4} \\
\hline \widehat{c}_{2} \\
\hline \widehat{c}_{3}
\end{array}\right) .
$$

We emphasize that the matrix is not explicitly reordered, but we factorize and solve the linear system in the order given in (3) as follows:

a) Initial off-line setup: As part of our off-line setup, we partially factorize the matrix such that $K=L D L^{\mathrm{T}}$, where

$L=\left(\begin{array}{c|c|c|c}L_{1,1} & 0 & 0 & 0 \\ \hline L_{2,1} & L_{2,2} & 0 & 0 \\ \hline 0 & 0 & I_{p} & 0 \\ \hline L_{4,1} & L_{4,2} & 0 & I_{p}\end{array}\right), \quad D=\left(\begin{array}{c|c|c|c}D_{11} & 0 & 0 & 0 \\ \hline 0 & D_{22} & 0 & 0 \\ \hline 0 & 0 & S^{-1} Z & I_{p} \\ \hline 0 & 0 & I_{p} & C\end{array}\right)$

For this partial factorization, the last 2-by-2 trailing blocks of $L$ and $D$ are not yet fully factorized, but will be factorized in the on-line factorization phase. Therefore, neither $L$ nor $D$ are yet in its respective full lower triangular nor diagonal form. We compute this partial $\mathrm{LDL}^{\mathrm{T}}$ factorization as follows:

1) We compute the $\operatorname{LDL}^{\mathrm{T}}$ factorization of $Q$ such that $Q=$ $L_{1,1} D_{1,1} L_{1,1}^{\mathrm{T}}$.

2) We compute the off-diagonal blocks $L_{2,1}$ and $L_{4,1}$ in the first block column of $L$ such that $L_{2,1}:=A\left(D_{1,1} L_{1,1}^{\mathrm{T}}\right)^{-1}$ and $L_{4,1}:=G\left(D_{1,1} L_{1,1}^{\mathrm{T}}\right)^{-1}$.
3) We compute the $\mathrm{LDL}^{\mathrm{T}}$ factorization of the second diagonal block $\widetilde{K}_{2,2}$ such that $\widetilde{K}_{2,2}=L_{2,2} D_{2,2} L_{2,2}^{\mathrm{T}}$, where $\widetilde{K}_{2,2}:=-\left(L_{2,1} D_{1,1} L_{2,1}^{\mathrm{T}}\right)$.

We put $\sim$ on top of the block to distinguish it from the corresponding block of the original matrix $K$.

4) We compute the off-diagonal block $L_{4,2}$ in the second block column of $L$ such that $L_{4,2}:=\widetilde{K}_{4,2}\left(D_{2,2} L_{2,2}^{\mathrm{T}}\right)^{-1}$, where $\widetilde{K}_{4,2}:=-\left(L_{4,1} D_{1,1} L_{2,1}^{\mathrm{T}}\right)$

5) We compute the last diagonal block $C$ of $D$ such that $C:=-\left(L_{4,1} D_{1,1} L_{4,1}^{\mathrm{T}}\right)-\left(L_{4,2} D_{2,2} L_{4,2}^{\mathrm{T}}\right)$.

It is not shown to simplify the notations, but the $\mathrm{LDL}^{\mathrm{T}}$ factorization of each diagonal block is computed with permutation.

b) On-line Factorization: At each iteration of the solve instance with a new $S^{-1} Z$, we can factorize the last two diagonal blocks of $D$ in (4), i.e.,

$\left(\begin{array}{c|c}S^{-1} Z & I_{p} \\ \hline I_{p} & C\end{array}\right)=\left(\begin{array}{c|c}I_{p} & 0 \\ \hline L_{4,3} & L_{4,4}\end{array}\right)\left(\begin{array}{c|c}D_{3,3} & \\ \hline & D_{4,4}\end{array}\right)\left(\begin{array}{c|c}I_{p} & L_{4,3}^{\mathrm{T}} \\ \hline & L_{4,4}^{\mathrm{T}}\end{array}\right)$

where

$$
\begin{cases}D_{3,3} & :=S^{-1} Z \\ L_{4,3} & :=D_{3,3}^{-1}=Z^{-1} S,\end{cases}
$$

and $\widetilde{C}=L_{4,4} D_{4,4} L_{4,4}^{\mathrm{T}}$ with $\widetilde{C}:=C-\left(Z^{-1} S\right)$. Since both $Z$ and $S$ are diagonal, the most computationally expensive part of the on-line factorization is the $\mathrm{LDL}^{\mathrm{T}}$ factorization of $\widetilde{C}$. In other words, our on-line factorization only factorizes a matrix of the dimension $p$, and this algorithm allows us to solve the realtime convex optimization where $p$, instead of $n+m+2 p$, is the largest dimension of the matrix that must be factorized within the realtime constraint.

In this letter, we only consider the QP problem, i.e., diagonal $S^{-1} Z$. However, the algorithm can be trivially extended to a more general case such as the second order cone programming (SOCP) [2], where $S^{-1} Z$ is replaced with a symmetric positive definite scaling matrix computed from the primal and dual variables (such as $S^{-1 / 2} Z S^{-1 / 2}$ ).

c) On-line Solve: With the complete $\mathrm{LDL}^{T}$ factorization of $K$, the solution to the linear system can be computed through the forward and backward substitutions.

\section{B. Fixed $Q, A$ and variable $G, S^{-1} Z$}

In some cases, the submatrix $G$ changes between the solve instances, but stays fixed during the solution iterations of one solve instance. In such cases, we introduce an intermediate off-line update step, where we only recompute the steps that use $G$. The cost of this intermediate step is amortized over all the solution iterations of the solve instance.

\section{a) Initial off-line setup:}

1) We compute the $\operatorname{LDL}^{\mathrm{T}}$ factorization of $Q$ such that $Q=$ $L_{1,1} D_{1,1} L_{1,1}^{\mathrm{T}}$.

2) We compute the off-diagonal block $L_{2,1}$ in the first block column of $L$ such that $L_{2,1}:=A\left(D_{1,1} L_{1,1}^{\mathrm{T}}\right)^{-1}$. 
TABLE I

NUMBER OF FLOATING POINT OPERATIONS NEEDED FOR FACTORIZATION.

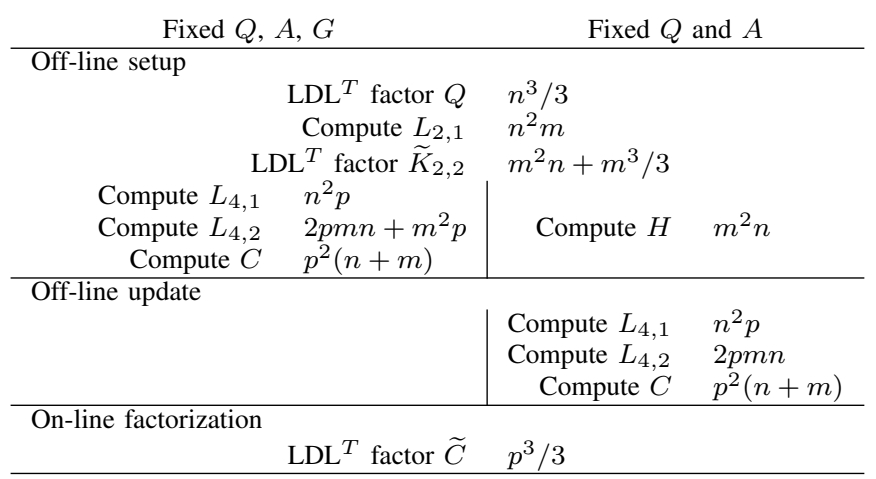

3) We factorize the second diagonal block $\widetilde{K}_{2,2}$ such that $\widetilde{K}_{2,2}=L_{2,2} D_{2,2} L_{2,2}^{\mathrm{T}}$, where $\widetilde{K}_{2,2}:=-\left(L_{2,1} D_{1,1} L_{2,1}\right)$

4) We partially compute the off-diagonal block in the second block column of $L$, i.e., $H:=-D_{1,1} L_{2,1}^{\mathrm{T}}\left(D_{2,2} L_{2,2}\right)^{-1}$.

b) Off-line update: To solve each convex optimization problem with a new submatrix $G$, we complete the off-line factorization as follows:

1) We compute the off-diagonal block $L_{4,1}$ in the first block column of $L$ such that $L_{4,1}:=G\left(D_{1,1} L_{1,1}^{\mathrm{T}}\right)^{-1}$.

2) We compute the off-diagonal block $L_{4,2}$ in the second block column of $L$ such that $L_{4,2}:=L_{4,1} H$.

3) We compute the last block $C$ of $D$ such that $C:=$ - $\left(L_{4,1} D_{1,1} L_{4,1}^{\mathrm{T}}\right)-\left(L_{4,2} D_{2,2} L_{4,2}^{\mathrm{T}}\right)$.

c) On-line Factorization: Finally, at each iteration with the new diagonal submatrix $S^{-1} Z$, we can cheaply perform the on-line factorization and solve as in Section $\amalg-\mathrm{A}$

To summarize our two structure-aware linear solvers, Table [] lists the required number of floating point operations at each step of factorization (in non-complex precision).

\section{IMPLEMENTATION}

For our experiments, we focused on the dense blocks $Q, A$, and $G$, and our implementation is based on LAPACK [14]. For example, to factorize the diagonal blocks, we used LAPACK's dsytrf subroutine that dynamically permutes the matrix to ensure the numerical stability. In contrast, the CVXGEN's generated solver uses static permutation and regularization to avoid the small diagonal entries through small diagonal shifts [6]. Compared to the dynamic pivoting, the static pivoting may be less stable or accurate, but it can be more efficient and leads to the fixed factorization time.

Unfortunately, LAPACK does not provide a flexible enough interface to take the full advantage of the symmetry in the KKT matrix. In particular, LAPACK has the subroutine dsytrs to apply $\left(L D L^{\mathrm{T}}\right)^{-1}$ using the $\mathrm{LDL}^{T}$ factorization computed by dsytrf, but it does not have a subroutine to apply $L^{-1}$ or $D^{-1}$ alone, which is needed, for example, at Step 2 of the off-line setup in Section II-A. Hence, we compute the nonsymmetric LDU factorization of the KKT matrix, $K=L D U$, where $L$ and $D$ have the same block structures as those in (4), and $U$ has the same structure as that of $L^{\mathrm{T}}$. With this implementation, at Step 1 of the initial off-line setup in Section [I-A. we let $L_{1,1}=I_{n}, D_{1,1}=I_{n}$, and $U_{1,1}=Q$, and then, we use dsytrf to compute the $\mathrm{LDL}^{\mathrm{T}}$ factorization of $Q$. Then, at Step 2, we compute, $L_{2,1}:=A U_{1,1}^{-1}$ and $L_{4,1}:=G U_{1,1}^{-1}$, while $U_{1,2}=A^{\mathrm{T}}$ and $U_{1,4}=G^{\mathrm{T}}$. We use LAPACK's dsytrs to apply $U_{1,1}^{-1}$ based on the $\mathrm{LDL}^{\mathrm{T}}$ factorization of $Q$.

Similary, at Steps 3, we set $L_{2,2}=I_{m}, D_{2,2}=I_{m}$, and $U_{2,2}=\widetilde{K}_{2,2}$, where $\widetilde{K}_{2,2}:=-\left(L_{2,1} U_{2,1}\right)$, and we factorize $\widetilde{K}_{2,2}$ using dsytrf. Then, at Step 4 , we compute, $L_{4,2}:=$ $-\left(L_{4,1} U_{1,2}\right) U_{2,2}^{-1}$ and $U_{2,4}:=L_{2,1} U_{1,4}$. Finally, at Step 5, we compute $C:=-\left(L_{4,1} U_{1,4}\right)-\left(L_{4,2} U_{2,4}\right)$.

\section{EXPERIMENTS}

We conducted all of our experiments on MacBook Pro ${ }^{\circledR}$, using just one core of $2.7 \mathrm{GHz}$ Intel ${ }^{\circledR}$ Core $^{\mathrm{TM}}$ i7. Our codes were compiled using gcc of Apple ${ }^{\circledR}$ LLVM version 5.1 with the optimization flag -Os. For our experiments, we linked our codes to BLAS and LAPACK provided in the Apple's Accelerate Framework [15], but on any other embedded system, it could be statically linked to open-source reference implementations of BLAS [16] and LAPACK [14].

\section{A. Results with fixed $Q, A, G$ and variable $S^{-1} Z$}

The performance results in Table $\Pi$ correspond to the largest convex optimization problem of the form (1), that CVXGEN could handle. The KKT matrix is of dimension 131, and the respective dimensions of the submatrices $Q, Z, G$, and $A$ are $95 \times 95,12 \times 12,95 \times 12$, and $95 \times 12$.

For this particular dimension of the matrix, the CVXGEN's generated solver was slower than calling LAPACK's nonsymmetric or symmetric solver, dgesv or dsysv, respectively, on the matrix $\widehat{K}$, even though LAPACK ignores the structure of the KKT matrix and performs dynamic pivoting.

Since LAPACK interface does not allow our off-line factorization to take full advantage of the symmetry of the KKT matrix, it was slower than the LAPACK's symmetric dsysv solver. However, it was faster than the LAPACK's non-symmetric dgesv solver or the CVXGEN's generated solver. More importantly, the cost of this initial off-line setup is amortized over all the solve instances, and by taking advantage of the fixed structure, our on-line factorization was significantly faster than the CVXGEN's factorization with speedups of about 131.3.

In Table [I] we also show the results when we consider the submatrix $Q$ to be diagonal. Since the submatrix $Q$ does not have to be factorized, compared to the case of a dense $Q$, our off-line factorization obtained a greater speedup over the CVXGEN's factorization. 
TABLE II

RUN TIME (IN SECONDS) FOR SOLVING KKT LINEAR SYSTEM.

\begin{tabular}{|c|c|c|}
\hline Technique & Factor & Solve \\
\hline CVXGEN & $5.25 \cdot 10^{-4}$ & $1.70 \cdot 10^{-5}$ \\
\hline LAPACK (dgesv) & $3.03 \cdot 10^{-4}$ & $2.20 \cdot 10^{-5}$ \\
\hline LAPACK (dsysv) & $1.78 \cdot 10^{-4}$ & $1.90 \cdot 10^{-5}$ \\
\hline $\begin{array}{l}\text { fixed } Q, A, G(\text { dense } Q) \text { : } \\
\text { initial off-line setup } \\
\text { on-line factor and solve }\end{array}$ & $\begin{array}{l}1.85 \cdot 10^{-4} \\
4.00 \cdot 10^{-6}\end{array}$ & $2.30 \cdot 10^{-5}$ \\
\hline $\begin{array}{l}\text { fixed } Q, A, G(\text { diagonal } Q) \text { : } \\
\text { initial off-line setup } \\
\text { on-line factor and solve }\end{array}$ & $\begin{array}{l}3.90 \cdot 10^{-5} \\
4.00 \cdot 10^{-6}\end{array}$ & $6.00 \cdot 10^{-6}$ \\
\hline $\begin{array}{l}\text { fixed } Q, A(\text { dense } Q) \text { : } \\
\text { initial off-line setup } \\
\text { off-line update } \\
\text { on-line factor and solve }\end{array}$ & $\begin{array}{l}1.21 \cdot 10^{-4} \\
7.00 \cdot 10^{-5} \\
4.00 \cdot 10^{-6}\end{array}$ & $2.30 \cdot 10^{-6}$ \\
\hline
\end{tabular}

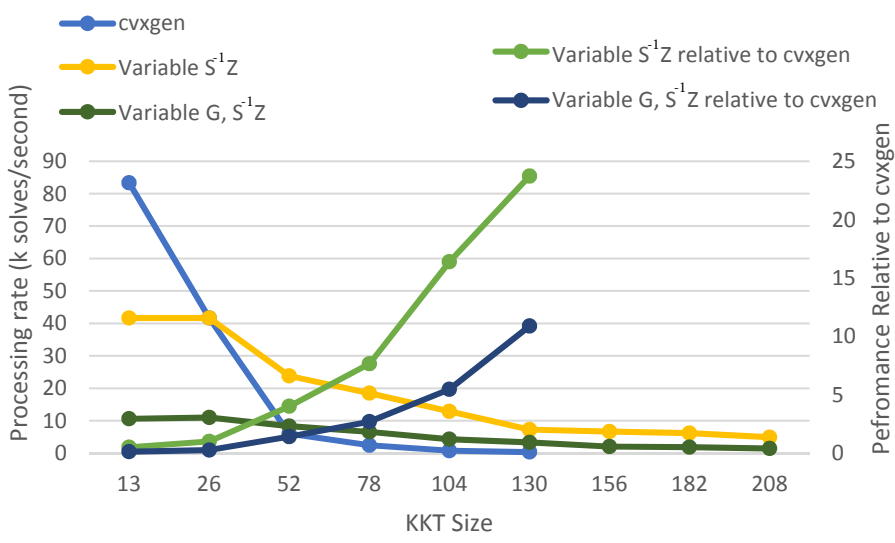

Fig. 1. Processing rate and relative performance with respect to CVXGEN

\section{B. Results with fixed $Q, A$ and variable $G, S^{-1} Z$}

The last few rows of Table II show the performance of our solver when only the submatrix $G$ changes between the instances of the solve. Even with the extra operations required for the non-symmetric factorization, by taking advantage of the structure, our on-line update was faster than the CVXGEN's factorization by a factor of about 7.5.

\section{Scaling with the problem size}

We compare the performance of our solver with that of CVXGEN, using a set of test problems of varying sizes; the KKT matrix of dimension from $13(n=10, m=1$, and $p=1)$ to $208(n=160, m=16$, and $p=16)$. Figure 1 plots the processing rates (the number of solves per seconds) and the relative performance of various schemes. For the KKT matrix with its dimension greater than $52(n=40, m=4$, and $p=4$ ), both of our solvers performed better than CVXGEN's. For problems with the matrix size larger than $130(n=100$, $m=10$, and $p=10$ ), CVXGEN could not generate the necessary code.

Figure 1 also shows that the relative performance of our two solvers, compared with CVXGEN, grows with the problem size, reaching to about 24 and 12 for the matrix size of 130 $(n=100, m=10$, and $p=10)$.

\section{CONCLUSION}

In this letter, we proposed techniques to reduce the time to factorize the KKT matrices for solving a realtime convex optimization on an embedded system. This technique takes advantage of the fact that many blocks of the KKT matrix do not change during the iterations of one solve instance. Our experimental results have shown that compared to the CVXGEN's generated solver, the factorization time can be reduced by two orders of magnitude.

\section{REFERENCES}

[1] D. P. Bertsekas, Convex Optimization Algorithms. Athena Scientific, Belmont, MA., 2015.

[2] S. Boyd and L. Vandenberghe, Convex optimization. Cambridge University Press, Cambridge, 2012.

[3] J. Mattingley and S. Boyd, "Realtime convex optimization in signal processing," IEEE Signal Processing Magazine, vol. 27, no. 3, pp. 5061, May 2010.

[4] D. Burns, W. Weiss, and M. Guay, "Realtime setpoint optimization with time-varying extremum seeking for vapor compression systems," in American Control Conference (ACC), 2015, July 2015, pp. 974-979.

[5] Y. Wang and S. P. Boyd, "Fast model predictive control using online optimization," in Proceedings World Congress of the International Federation of Automatic Control (IFAC), Jejudo Island, Korea, June 2001, pp. 6974-6997.

[6] J. Mattingley and S. Boyd, "CVXGEN - code generation for convex optimization," Optimization and Engineering, vol. 13, no. 1, pp. 1-27, March 2012. [Online]. Available: http://http://cvxgen.com/

[7] A. Domahidi, E. Chu, and S. Boyd, "ECOS: An SOCP solver for embedded systems," in European Control Conference (ECC), Zurich, Switzerland, July 2013, pp. 3071-3076. [Online]. Available: https://www.embotech.com/ECOS

[8] A. Stojanov, G. Ofenbeck, T. Rompf, and M. Püschel, "Abstracting vector architectures in library generators: Case study convolution filters," in ACM International Workshop on Libraries, Languages and Compilers for Array Programming (ARRAY), 2014, p. 14.

[9] M. Grant, S. Boyd, and Y. Ye, "Disciplined convex programming," in Global Optimization: From Theory to Implementation (Nonconvex Optimization and Its Applications), L. Liberti and N. Maculan, Eds. Springer Science and Business Media, 2006, pp. 155-210.

[10] M. Grant and S. Boyd, "CVX: matlab software for disciplined convex programming version 2.1," 2015. [Online]. Available: http: //cvxr.com/cvx

[11] J. Nocedal and S. Wright, Numerical Optimization, 2nd ed. Springer Verlag, 2006.

[12] W. H. Press, S. A. Teukolsky, W. T. Vetterling, and B. P. Flannery, Numerical Recipes in C: The Art of Scientific Computing, 3rd Ed. Cambridge University Press, Cambridge, 20007.

[13] J. Mattingley and S. Boyd, "Automatic code generation for real-time convex optimization," in Convex Optimization in Signal Processing and Communications, D. P. Palomar and Y. C. Eldar, Eds. Cambridge: Cambridge University Press, 2009, ch. 1, pp. 1-41.

[14] "APACKLinear Algebra PACKage," 2016. [Online]. Available: http: //www.netlib.org/lapack/

[15] Apple Inc, “Accelerate Framework," 2016. [Online]. Available: https://developer.apple.com/reference/accelerate

[16] "BLAS Basic Linear Algebra Subprograms," 2012. [Online]. Available: http://www.netlib.org/blas/ 
Dear Prof. Nooshabadi:Manuscript ID IEEE-ESL-Jan-17-0002.R1 entitled "Structure-aware Linear Solver for Realtime Convex Optimization for Embedded Systems" submitted to the Embedded Systems Letters, has been reviewed. The comments of the reviewer(s) are included at the bottom of this letter.

The reviewer(s) have recommended publication, but also suggest some minor revisions to your manuscript. Therefore, I invite you to respond to the reviewer(s)' comments and revise your manuscript promptly.

To revise your manuscript, log into https://mc.manuscriptcentral.com/les-ieee and enter your Author Center, where you will find your manuscript title listed under "Manuscripts with Decisions." Under "Actions," click on "Create a Revision." Your manuscript number has been appended to denote a revision.

You will be unable to make your revisions on the originally submitted version of the manuscript. Instead, revise your manuscript using a word processing program and save it on your computer. Please also highlight the changes to your manuscript within the document by using the track changes mode in MS Word or by using bold or colored text.

Once the revised manuscript is prepared, you can upload it and submit it through your Author Center.

When submitting your revised manuscript, you will be able to respond to the comments made by the reviewer(s) in the space provided. You can use this space to document any changes you make to the original manuscript. In order to expedite the processing of the revised manuscript, please be as specific as possible in your response to the reviewer(s).

IMPORTANT: Your original files are available to you when you upload your revised manuscript. Please delete any redundant files before completing the submission.

Because we are trying to facilitate timely publication of manuscripts submitted to the Embedded Systems Letters, your revised manuscript should be uploaded as soon as possible but no later than two weeks from today. If it is not possible for you to submit your revision in this period, we may have to consider your paper as a new submission.

Once again, thank you for submitting your manuscript to the Embedded Systems Letters and I look forward to receiving your revision.

Sincerely,

Prof. Sri Parameswaran

Editor in Chief, Embedded Systems Letters

sridevan@cse.unsw.edu.au

AE Comments to Author:

Associate Editor: Gordon-Ross, Ann

Comments to the Author:

Even though one of the reviewers suggests accept as is, there is still a few concerns that the authors should address in a minor revision. One reviewer mentions a comment about "Page 2, column 2, line 15" that is important to address before acceptance.

The word "cheaply" has been dropped!

Reviewer(s)' Comments to Author: 
Reviewer: 1

Comments to the Author

Page 1, column 1, line 4. "Liner solver"

Corrected!

Page 1, column 2, line 20. "the following function". (1) is an optimization problem, not a function.

Corrected!

Page 2, column 1, line 21. It would be useful to indicate how exactly Ktilde is regularized. I assume a multiple of the identity is added to the 11 and 22 blocks in (2), and subtracted from the 33 and 44 blocks.

The reviewer assumption is almost correct. However, instead of describing a standard well-known technique, we have provided a reference (Ref [11]) where the procedure is described in details.

Page 2, column 2, line 15. "we can cheaply factorize". It should be kept in mind that $\mathrm{p}$ is often greater than $\mathrm{n}$, so an order $\mathrm{p}^{\wedge} 3$ complexity is not cheap. In fact, in a standard QP solver, one would normally reduce the KKT system to one containing the matrix $Q+G^{\wedge} T{ }^{*} S * Z^{\wedge}-1{ }^{*} G$. In a problem with no equality constraints this has complexity $p^{*} n^{\wedge} 2$ for computing the matrix and $n^{\wedge} 3$ for factorizing it.

The word "cheaply" has been dropped!

Page 2, column 2, line 37. "where $S^{\wedge}-1^{*} Z$ is of the symmetric form $S^{\wedge}\{-1 / 2\}^{*} Z^{*} S^{\wedge}\{-1 / 2\}^{\prime}$. I still find this a very strange and almost absurd formulation. You could say that $S^{\wedge}-1{ }^{*} Z$ is replaced by a matrix $S^{\wedge}\{-1 / 2\}^{*} Z^{*} S^{\wedge}\{-$ $1 / 2\}$, but that only makes sense if you also define $S$ and $Z$. Since the details are not important here, it is sufficient to say that $S^{\wedge}\{-1\}{ }^{*} Z$ is replaced with a symmetric positive definite scaling matrix computed form the primal and dual variables.

We agree with the reviewer. We have replaced the wording with "where $\$ S^{\wedge}\{-1\} Z \$$ is replaced with a symmetric positive definite scaling matrix computed from the primal and dual variables (such as \$S^\{-1/2\}ZS^\{-1/2\}\$)"

Page 3, column 2, line 37. How are the problem data chosen? Are they randomly generated?

Yes, they are generated randomly!

Page 3, column 2, line 44, "LAPACK ... perform dynamic pivoting"

Corrected!

Page 4, column 1, line 47. "KTT matrix"

Corrected!

Reviewer: 2

Comments to the Author

It is important to correct the following: 
Page 1, first column, lines 46-53, there are 2 wrong statements. ECOS as such is not a code generation system. It is a standalone solver. There is a code generation system for ECOS but that is not reference [7]. Second, DCP as such has nothing to do with code generation either. It is for checking convexity of functions and sets. The statements are misleading and should be corrected.

To avoid confusion the sentences are reworded as:

"tools such as CVXGEN code generator [6] and ECOS [7] provide frameworks for realtime convex optimization solvers."

"CVXGEN takes a high-level description of the optimization problem, employs the CVX technique of disciplined convex programming (DCP) [9] to ensure convexity, and then generates a flat, and libraryfree C code."

Page 2, first column, line 2, the adaptive filtering design problem is not referenced. Unclear to which problem exactly the authors refer.

Ref [3] has been added.

Page 32, second column, line 38: the expression $S^{\wedge}\{-1 / 2\} Z S^{\wedge}\{-1 / 2\}$ might not be diagonal for some scaling matrices such as the popular Nesterov-Todd scaling. If this is a limitation the authors should mention it.

We have not made a claim about the $S^{\wedge}\{-1 / 2\} Z S^{\wedge}\{-1 / 2\}$ being diagonal. We stated that it is symmetric. 